\title{
La Disforia de género y suicidio en tiempos de crisis sanitaria
}

\author{
Gender dysphoria and suicide in times of health crisis
}

\author{
Calero Romoleroux María Paula \\ Universidad Técnica de Machala \\ mcalero2@utmachala.edu.ec \\ Machala - Ecuador \\ Villavicencio-Aguilar Carmita \\ Universidad Técnica de Machala \\ cvillavicencio@utmachala.edu.ec \\ Machala - Ecuador
}

\section{Resumen}

Este estudio busca constatar la presencia de riesgo suicida en personas con disforia de género durante la crisis sanitaria COVID-19. Desde un enfoque cualitativo se realizó una entrevista semiestructurada a una persona transgénero en la ciudad de Machala. Se identificó la presencia de sintomatología ansiosa-depresiva, ideación suicida y actos autolíticos que se incrementaron durante la crisis sanitaria, a través de la revisión de un análisis de caso de joven transgénero. Los pacientes con disforia de género tienen un riesgo significativo de comorbilidad con intentos suicidas. A partir de la aplicación de esta investigación se realizarán posteriores protocolos estandarizados dirigidos a los profesionales de la salud, centrados en la necesidad de orientación, apoyo y evaluación de riesgo que estas personas requieren. De hecho, un enfoque colaborativo y multidisciplinario puede ayudar a cuidar a estas personas en situación de vulnerabilidad que pertenezcan a grupos minoritarios y evitar resultados adversos.

Palabras Claves: Disforia de género, Crisis sanitaria, Suicidio, Transgénero. 


\section{Abstract}

This study seeks to verify the presence of suicidal risk in people with gender dysphoria during the COVID-19 health crisis. From a qualitative approach, a semi-structured interview was conducted with a transgender person in the city of Machala. The presence of anxietydepressive symptoms, suicidal ideation and autolytic acts that increased during the health crisis were identified through the review of a case analysis of a young transgender. Patients with gender dysphoria have a wilde risk of comorbidity with attempts suicidal. Based on the application of this research, subsequent standardized protocols aimed at health professionals will be carried out, focused on the need for guidance, support and risk assessment that these people require. In fact, a collaborative and multidisciplinary approach can help to care for these vulnerable people who belong to minority groups and avoid adverse outcomes.

Key Words: Gender dysphoria, Health crisis, Suicide, Transgender.

\section{Introducción}

La crisis sanitaria de COVID-19 y sus múltiples efectos han perjudicado la salud mental de muchas personas, en específico para aquellas que ya padecían enfermedades mentales o trastornos, debilitando sus fortalezas o acelerando su sintomatología. Esto es especialmente cierto para las personas con disforia de género, quienes experimentan mayores disparidades de salud en comparación con las poblaciones heterosexuales y cisgénero (Su et al., 2016). Por ello, el presente trabajo tiene como finalidad constatar la presencia de riesgo suicida y sus resultados adversos que se incrementaron durante la crisis sanitaria a través de la revisión de un análisis de caso de joven transgénero.

En ese contexto, se debe recabar información relevante sobre la disforia de género, desde sus inicios hasta la actualidad, para una asertiva comprensión y evaluación del caso. La disforia de género aparece en 1869 a partir de la publicación de un artículo de Westphal denominado "Die contrare sexualemp-finding", se expone detalles sobre el caso de una mujer a quien se realiza una antropometría y quien expresaba su inconformidad con su sexo anatómico y roles establecidos y deseaba cambios al respecto, inclusive desde temprana edad evidenciaba atracción por otras niñas y en su adultez describe diferentes prácticas sexuales con otras mujeres (Crozier, 2008).

Eventualmente, Foucault designaría aquel momento como la fecha de nacimiento del "homosexual" (Foucault, 2010). Sin embargo, Hirschfeld (1910) citado en Balzer (2011) en aquella época ostentaba su teoría denominada "tercer sexo", planteaba que poseer una adecuada comprensión científica de la homosexualidad prescindiría la hostilidad dirigida hacia este grupo minoritario. Por tanto, este mismo psicólogo en 1910 publica e introduce el término "travestidos" que en la modernidad hace referencia a la identidad transexual y travesti vinculados con la feminidad-masculinidad (Lorenci, 2013). 
En ese sentido, es relevante citar a Cauldwell (1949) autor que es el primero en hacer uso de la terminología transexual describiéndola como:

Cuando un individuo que está desfavorablemente afectado psicológicamente determina vivir y presentarse como miembro del sexo al que no pertenece, este individuo se puede llamar psicópata transexual. Significa, simplemente, que no está sano mentalmente, y por esto esa persona desea vivir en el sexo opuesto. (p. 275)

Desde esa perspectiva surgió un hecho que cautivo una curiosidad considerable por parte de la población, que se conoce en la historia como la primera intervención de "la cirugía de reasignación de sexo" realizada en 1952 (Chárriez, 2013). A continuación, el sexólogo Benjamín notifica de un caso de una mujer asignada en el cuerpo de un hombre, a partir del cual este autor explicó el significado de "transexualismo" como la relación existente entre el sexo biológico y la certeza de integrar parte del otro sexo, y que con ello el individuo desea iniciar un proceso de cambio (Benjamín, 1953).

Así mismo, Money et al. (1984) citados en Swaab (2004) analizaron la conciencia de neutralidad a partir del nacimiento y respaldó la concepción de género en 1957 postulando que los infantes forjan la diferencia entre ser mujer y hombre a consecuencia de las secuelas de las prácticas sociales y experiencias previas. A partir de entonces, según Orozco-Calderón (2011) la discordancia entre "el sexo anatómico y la identidad de género" se rotuló como la condición psicopatológica del "trastorno de la identidad de género".

La American Psychiatric Association (APA) utilizó para su nombre diagnóstico cuando se introdujo por primera vez en el DSM-III (APA, 1980). Sin embargo, tras diversos cuestionamientos en el DSM-III - $\mathrm{R}$ se clasificó en tres tipos: transexualismo, no transexualismo y no especificado de otra manera (APA, 1987). Luego los dos primeros tipos se combinaron y se estandarizaron posteriormente en el nombre oficial de diagnóstico de "trastorno de identidad de género" en el DSM-IV-TR (APA, 2000).

En ese sentido, se generaron algunas demandas favorables en referencia a los derechos del colectivo trans como Comisario Europeo de Derechos Humanos (2009) y del Parlamento Europeo (2011) al considerar que aquel término era discriminatorio. Por ello, se realizó un cambio remplazando la terminología de "trastorno de identidad de género" por disforia de género (GD) en una tentativa de referir mejor "el malestar clínicamente significativo" que sienten las personas cuyo sentido de ser hombre o mujer difiere del género que se les asignó al nacer en función de su sexo (APA, 2014).

Algunos autores consideran que esta etiqueta diagnóstica es más consistente con la terminología en relación a la sexología clínica y elimina la connotación de que las personas con inconformidad de género están "desordenados" a nivel mental, aunque esta constante discusión a la que se refiere Dellacasa (2017) existe a raíz de la adherencia de ubicar la condición trans en el plano de la patología.

En el mejor de los casos, esta connotación toma relevancia al otorgarle su propio capítulo distinto de "Disfunciones sexuales y trastornos parafílicos", con el propósito de realizar un 
adecuado diagnóstico diferencial para determinar con mayor seguridad que las personas puedan recibir beneficios del tratamiento de reasignación de sexo, ya sea quirúrgico u hormonal (AETSA, 2014).

De hecho, la APA (2017) alude que el diagnóstico de disforia de género podría generar una secuela estigmatizadora, pero explica su representación certificando que se genera un mejor acceso a la atención clínica y cobertura clínica en beneficio de la salud mental; para la Organización Panamericana de la Salud (OPS) significa no colocar un distintivo permanente en las personas (OPS, 2012). En otras palabras, se determinó que el problema no es el transgenerismo en sí, sino los desórdenes que se generan en las personas trans a raíz de la respuesta de una sociedad que las discrimina.

Por el contrario, la alocución de la comunidad transgénero se basa en que nacieron así y no fue su elección serlo (Serret, 2009). Por consiguiente, rechazan el hecho de ser estas personas quienes tienen que buscar ayuda o tratamiento con un profesional y enfatizan sobre su deseo de fomentar la aceptación social.

Es así que, se han realizado varios intentos de animar la inclusión de las personas transgénero, que se puede pensar que han dado fruto. No obstante, para López (2018), aún permanecen vigentes errores heteronormativos como la religión, el estado, la familia, entre otras costumbres y pensamientos que influyen al entendimiento y comprensión de diversidad de género como tal. Entre las concepciones más arraigadas se encuentran los que tradicionalmente se muestran de acuerdo con el planteamiento de que solo hay dos seres sexuados como el hombre y la mujer, que la orientación del deseo perennemente va ser heterosexual, que los roles sexuales y la identidad social corresponderán como femenino y masculino y que cada uno concernirá de acuerdo al sexo, y por el contrario se lo supone como un acto "inmoral" o se lo relaciona con un trastorno mental.

A propósito de estos errores, la continuidad de los mismos de generación en generación, ha originado mayor discriminación y exclusión donde los transexuales, exteriorizan emociones negativas y sintomatología ansiosa por anhelar corresponder a esta sociedad heteronormativa. El estigma social genera sentimientos de culpa y vergüenza con consecuencias de baja autoestima y sentimientos de no merecer el amor de los demás, con mayor desventaja para las personas trans desempleadas o con bajos ingresos que podrían percibir mayor rechazo, maltrato o ser sujetos de discriminación social (OPS, 2012).

Efectivamente, Napiarkorvski, (2012) cita que las personas trans constituyen una población concretamente vulnerable debido a que "se han encontrado permanentemente con la intolerancia, la agresividad, la humillación y la marginación” (p.221), padeciendo altos niveles de estrés, discriminación laboral, estigmatización, enjuiciamiento y a veces violencia, por parte de la sociedad, lo que contribuye de forma directa y significativa a su vulnerabilidad que se asociada a depresión, ansiedad y tendencias o conductas suicidas (pensamientos e intentos), son frecuentes, y se asocian con el estigma y la discriminación (Bockting et al., 2011). 
Las manifestaciones de la ideación suicida se deben de distinguir de la ansiedad y la depresión, se evalúa el riesgo de suicidio: Ideación, uso de sustancias, falta de metas, angustia, desaliento, aislamiento, ira, aturdimiento y cambios en el estado de ánimo y humor (OPS, 2012); estos signos de riesgo de suicidio se apoyan en hablar, escribir o amenazar con quitarse la vida, planeación suicida (formas de atentar), e historial de intento suicida.

En tiempos de emergencia sanitaria (COVID-19) las personas trans son más vulnerables a las complicaciones de salud física y psicológica, en relación con la población general, tenían seis veces mayor emergencia de atención por ansiedad o trastorno del estado de ánimo, tres veces más posibilidades de usar medicina (antidepresivos y ansiolíticos), y seis veces más probabilidades de haber sido hospitalizados después de un intento de suicidio (Bränström y Pachankis, 2019). Es crucial una evaluación de salud mental para identificar a las personas con disforia de género, en especial evaluaciones psicológicas, por el alto riesgo de desarrollar serios desajustes emocionales e intentos suicidas (Pan et al., 2020).

En esa línea, en medio de la pandemia se evidenciaron diversas desigualdades, entre ellas la de género, por ello esta investigación busca constatar la presencia de riesgo suicida en personas con disforia de género durante la crisis sanitaria COVID-19.

\section{Materiales y Métodos}

El método de investigación cualitativa utilizada en este análisis es fenomenológico interpretativo (IPA). Las raíces de la IPA parten de la hermenéutica (Smith, et al., 2009). Esta metodología IPA busca recabar la descripción detallada y el caudal de la experiencia vivida por el participante mediante la revelación de temáticas centrales entre las investigadorasentrevistado, en cierta medida están involucrados en el proceso interpretativo como un componente clave de nivel realista, se ha utilizado principalmente para investigaciones psicológicas (Williams et al., 2010).

Se obtuvo la colaboración de la entrevista a través de una persona activista de la comunidad trans relacionada con el sujeto entrevistado. El contacto fue vía telefónica y después de comunicar el objetivo del trabajo, proporcionó la información de contacto; posteriormente se tomó contacto telefónico, se explicó el objetivo y el proceso, al conocerlo vía telefónica consintió en participar, se concertó una reunión vía virtual en un horario de comodidad para el colaborador, un único caso y al final referenciando la situación de sus amistades trans.

Los temas que se plantearon fueron direccionados en las preguntas de las entrevistas, centradas principalmente en las descripciones / experiencias: de tratamiento hormonal, sentimientos, situaciones del participante durante la emergencia; tanto con los sistemas de apoyo formales e informales. La entrevista individual vía telefónica y virtual se llevó a cabo dentro de un período de dos semanas. Los diálogos duraron entre 90 y 120 minutos; estaban en cinta de audio y video y transcritas textualmente. Las notas de campo se tomaron 
inmediatamente después de cada entrevista, que resume las principales impresiones y conceptos o temas que se trataron en la próxima entrevista.

Los datos se analizaron de acuerdo con procedimiento IPA (Smith, et al. 2009). Se leyó y se releyó las transcripciones, seguidas de notas interpretativas escritas en el margen izquierdo de la transcripción. Se hicieron notas, percepciones, y los resúmenes de cada entrevista. Siguiendo este procedimiento, se volvió a los datos y se hizo la identificación de temas que se observaron en el margen derecho de las transcripciones. Una lista de temas fue producida y citas correspondientes por cada tema. Se reconceptualizaron, omitieron o se fusionaron con otra temática paralela, se buscó similitudes y diferencias dentro de los temas. Se utilizó la notación de transcripción: ". . ." en las citas para indicar que el material fue omitido para acortar cita.

Con el fin de asegurar la confidencialidad de la información que pueda revelar la identidad del entrevistado en relación con temas sensibles fue contactado por teléfono e informado de la investigación. Se organizó una reunión de seguimiento telefónico, En la llamada de seguimiento, fue aprobada verbalmente la entrevista para su publicación y comentó sobre la interpretación de la entrevista. Para mejorar la validez de las interpretaciones, las autoras verificaron en relación con las transcripciones, elección de los temas emergentes, extractos elegido para ilustrar las experiencias del participante.

\section{Resultados y Discusión}

Cuatro temas se obtuvieron mediante el análisis interpretativo de la entrevista. Los temas son: (1) yo y mi familia; (2) salud, estado anímico; (3) proceso médico; y 4) Impacto psicológico y apoyo social en la comunidad trans. Las descripciones de cada tema se presentan junto con citas textuales de la persona entrevistada.

\section{Tema 1 articular: Yo, mi familia}

El entrevistado identifica su sentir con respecto a su identidad de género, durante la entrevista se muestra al inicio un poco de cautela y busca cuidadosamente las palabras para contestar, se observa así mismo en forma positiva "Un chico alegre, carismático, amiguero, me gusta llevar la calma y sin conflictos". Se define como representante de su comunidad trans, aunque considera que está en proceso de alcanzar su meta, aún persisten rasgos de inseguridad y falta de comunicación familiar. "Soy la reina de la comunidad trans, pero me considero transformista en proceso de transición y tratamiento hormonal."

Manifiesta que a los 6 años notó que su identidad sexual era distinta al resto de niños de su grupo etario, expresión de inconformidad con su sexo anatómico y roles establecidos y deseos de cambio desde temprana edad (Crozier, 2008); considera que el mejor cambio en su vida habría sido "Haber nacido mujer", existe la necesidad de sentirse con deseo de otro sexo diferente (APA, 2014). 
Al principio no tuvo apoyo de su familia nuclear, con el paso del tiempo sus padres aceptaron su decisión y la "toleran", sin embargo, aún le cuesta tomar riesgos y grandes decisiones debido a su falta de seguridad. La comunicación familiar aún la considera poco óptima como le agradaría que fuera, especialmente con su hermano menor. "Yo me di cuenta a temprana edad que era diferente, desde que estaba en la escuela a partir de segundo año de básica. "Sí, me considero una persona segura, pero en ocasiones me es difícil tomar ciertas decisiones"; ...para mis familiares fue un poco duro, tuve que irme de casa porque mis padres no me aceptaban en principio, pero mis abuelos maternos me ayudaron y me apoyaron en mi decisión. Luego con el tiempo mis padres lo aceptaron y toleran”.

\section{Tema 2 articular: salud, estado anímico}

Señala que durante el periodo de confinamiento por la crisis sanitaria experimentó situaciones fisiológicas relacionadas con su estado de salud por desarrollar el Covid 19 y su estado físico (Infección bronquial, aumento de peso, poco ejercicio físico y alteración del sueño), experimentan mayores contrastes de salud en comparación al resto de la población (Su et al., 2016). "Me afecto el hecho que tuve el virus, y mi salud se vio afectada. En mi caso me afectó en apetito porque aumenté un poco de peso, porque no podía hacer mi rutina de ejercicios a la que estaba acostumbrado. Al inicio de la pandemia...estaba pendiente de las noticias y de las redes sociales, y los contenidos que visualice me afectaron en mi rutina de sueño"

El estado emocional es el más afectado por haberse truncado sus planes futuros, la situación financiera para su tratamiento y reasignación de sexo ha desarrollado múltiples emociones y sentimientos negativos, expresados en llanto, tristeza, preocupación, aburrimiento, frustración, presentan seis veces por ansiedad o trastorno del estado de ánimo, tres veces más posibilidades de usar medicina (antidepresivos y ansiolíticos) (Bränström y Pachankis, 2019). "Me sentí un poco frustrado", en ocasiones con pocas ganas de realizar alguna actividad y aburrido"; "...durante la pandemia tuve que gastar mis ahorros ya que no me encontraba laborando y eso me causó malestar porque no sabía que iba a pasar a futuro”; “...me preocupaba cuando iba a poder trabajar, el lugar donde trabajaba cerró por motivo de pandemia”.

La depresión y la ideación suicida estuvieron presentes en especial por la preocupación económica por el gasto de sus ahorros para la reasignación de sexo. Exhiben seis veces más probabilidades de haber sido hospitalizados después de un intento de suicidio (Napiarkorvski, 2012; Bränström y Pachankis, 2019). "La parte económica me afectó porque yo estaba ahorrando para mi cirugía de reasignación de sexo y el hecho de no salir, no poder comunicarse con sus seres queridos”. “...Me sentí un poco deprimido, porque ya hice planes y fue algo de improvisto. ...Tuve pensamientos negativos que hicieron pensar en el suicidio, pero traté de llamar a amistades y me brindaron palabras de apoyo y estos pensamientos no pasaron a mayores."

\section{Tema 3: proceso médico}

En relación a la atención médica en tiempos de pandemia, los procesos se han suspendido para atender la emergencia y esto ha traído consigo que el tratamiento en salud mental se haya 
sufrido una interrupción, provocando sentimientos de angustia. La evaluación psicológica es crucial por el alto riesgo de desarrollar serios desajustes emocionales (Pan et al., 2020). "Sentía intranquilidad porque no pude seguir con mi tratamiento hormonal, me generó una autoestima baja, porque tenía planes y la pandemia me retrasó, porque prácticamente 8 meses estuve sin trabajo y eso me afectó en la parte psicológica. Me encontraba en tratamiento psicológico antes de la pandemia, pero me lo suspendieron porque se les dio prioridad a las personas que llegaban con síntomas de Covid-19”.

\section{Tema 4: Impacto psicológico y apoyo social en la comunidad trans}

Con respecto al impacto psicológico en la comunidad trans, el participante comenta sobre la afectación que padecieron sus integrantes por el estigma social y las actividades a las que se dedicaban en el área de eventos sociales, por el confinamiento no se producían y por lo tanto, no había ingresos económicos, como consecuencia se liberaron altos niveles de estrés, por discriminación laboral y estigmatización a los que estaban expuestos y ligados a la depresión, ansiedad (Bockting et al., 2011; Napiarkorvski, 2012; OPS, 2012). " Dentro de mi comunidad algunas se vieron muy afectadas por la cuestión laboral por la discriminación, y personas que dependían de la belleza, decoración de eventos, no había eventos, ni actividades que realizar. Así que les afectó en gran medida, porque su trabajo era diario y no tenían ingresos. La mayoría de compañeras son personas que tienen que pagar arriendo porque viven solas".

La economía también fue a fuente de ansiedad psicológica, depresión y situaciones de conductas autolíticas; con tendencias hacia pensamientos e intentossuicidas (Napiarkorvski, 2012). "En relación a mis compañeras, estaban preocupadas con las deudas a bancos y estaban colapsadas, comentaron que se encontraban deprimidas”; “...supe de un caso donde una amiga llegó a cortarse las venas”,

La ayuda social local o nacional no hizo presencia, recurrieron a activistas de grupos minoritarios para que consigan gestionar ayuda internacional para cuestiones básicas por el desmedro de su economía. "Se necesitaba ayuda y realizar trámites para obtener ayuda de asociaciones internacionales. Si recibimos ayuda, pero tuvimos que buscar la manera, a través de activistas para que unas organizaciones internacionales nos otorgaran un bono, alimentos, o kits de aseo para la comunidad trans".

\section{Conclusiones}

La crisis sanitaria del Covid-19 implicó afectaciones severas en varios aspectos y de manera transcendental en la salud mental de los individuos a nivel mundial. A raíz de ello se agravaron diversos trastornos mentales. En este caso el trastorno de disforia de género, y en base a los análisis expuestos se establece que los pacientes con disforia de género tienen un riesgo significativo de comorbilidad con posibles intentos suicidas que se acrecentaron durante la crisis sanitaria. Un ejemplo de ello, es el caso de joven transgénero mostrado, a través del cual se logró comprobar la presencia de sintomatología ansiosa-depresiva, ideación 
suicida y actos autolíticos que se desencadenaron e incrementaron durante el estado de salud emergente que por su singularidad impidió el acceso a una atención inmediata en los servicios de salud pública, y aún más grave se restringió el apoyo psicológico, generando una mayor inestabilidad emocional y aumentando más aún la probabilidad de vulnerabilidad que preexiste al formar parte de la comunidad trans.

Mediante esta investigación se hace un llamado a tomar en consideración a la comunidad trans para futuras investigaciones en la rama de la Psicología Clínica, puesto que al tener mayor probabilidad de discriminación y no aceptación social se impide su bienestar psicológico, y al no ser atendidos de manera asertiva, ni oportuna se puedan desarrollar desajustes emocionales en los involucrados. De hecho, un enfoque colaborativo y multidisciplinario puede ayudar a cuidar a estas personas en situación de vulnerabilidad que pertenezcan a grupos minoritarios y evitar resultados adversos para ellos.

Cabe recalcar que la principal limitante es el tamaño de la muestra, a futuro es importante ampliar el número de casos para extender los resultados a adquirirse de manera más óptima y sin sesgos. Así mismo, a partir de la aplicación de esta investigación se recomienda en futuras líneas de investigación se desarrollen protocolos estandarizados dirigidos a los profesionales de la salud, centrados en la necesidad de orientación, apoyo y evaluación de riesgo que estas personas requieren.

\section{Referencias}

American Psychiatric Association. (1980), Diagnostic and statistical manual of mental disorders (3a ed.)(DSM-III), Washington, D.C., American Psychiatric Association (trad. Cast. En Barcelona, Masson, 1984).

American Psychiatric Association. (1987), Diagnostic and statistical manual of mental disorders (3 ${ }^{a}$ ed.revisada) (DSM-III-R), Washington, D.C., American Psychiatric Association (trad. Cast. En Barcelona, Masson, 1988).

American Psychiatric Association. (2000). Diagnostic and statistical manual of mental disorders, (4 th ed., text revision). Washington, DC: American Psychiatric.

American Psychiatric Association. (2014). Diagnostic and statistical manual of mental disorders: DSM-5. Arlington, VA Washington, D.C: American Psychiatric Association.

American Psychiatric Association. (2017). DSM-5 Fact Sheets.

Balzer, C. (2011). "Eu acho transexual e aquele que disse: eu sou transexual" reflexiones etnológicas sobre la medicalización globalizada de las identidades trans a través del ejemplo de brasil. In M.Missé \& G. Coll -

Planas (Eds.), El género desordenado. Críticas en torno a la patologización de la transexualidad (pp. 81-96). Barcelona: Egales.

Benjamin H. (1953). Travestismo y transexualismo. Revista Internacional de Sexología. 153, 391- 396. 
Bockting, W., Miner, M., Swinburne, R., Hamilton, R., \& Coleman, E. (2013). Stigma, mental health, and resilience among an online sample of the U.S. transgender population. Journal of Public Health, 103(5), 943-951. doi:10.2105/AJPH.2013.301241

Bränström, R., \& Pachankis, J. (2019). Reduction in mental health treatment utilization among transgender individuals after gender-affirming surgeries: A total. European Journal of Public Health, 29(4), 174-181. doi:10.1093/eurpub/ckz185.465

Cauldwell, D. O. (1949). "Psychopatia transexualis." Sexology, 16, 274-80.

Chárriez, M. (2013). La transexualidad: ¿construcción de una identidad? Revista Griot, 6(1), 18-28.

Comisario Europeo de Derechos Humanos (2009) "Human Rights and Gender Identity". Strasbourg. Disponible online en: https://rm.coe.int/16806da753

Crozier, I. (2008). Pillow Talk: credibilidad, confianza y la historia del caso sexológico. Hist. Sci., 48, 375-404. https://doi.org/10.1177/007327530804600401

Dellacasa, M. A. (2017). Una mirada arqueológica de los discursos sobre transexualidad: modalidades de producción de conocimiento y subjetividades. Psicoperspectivas, 16(3), 17-28. https://doi.org/10.5027/psicoperspectivas-vol16-issue3-fulltext-1053

Foucault, M. (2010). Historia de la sexualidad. La voluntad del saber. (2 ${ }^{\mathrm{a}}$ ed.) (vols. 1) Aires: Siglo XXI Editores.

Lopez, F. (2018). Disforia de género en la infancia y adolescencia. Revista de Formación Continuada de la Sociedad Española de Medicina de la Adolescencia, 11.

Lorenci, M. (2013). Repensando los orígenes de la disforia de género. Revista de Estudios de Juventud, 33-50.

Martínez, I. (2014). Disforia de género. Síntesis de evidencia sobre aspectos relacionados con diagnóstico, tratamiento y resultados en salud. - Sevilla: Agencia de Evaluación de Tecnologías Sanitarias.

Napiarkorvski, Federico (2012). Vulnerabilidad de derechos en personas trans. IV Congreso Internacional de Investigación y Práctica Profesional en Psicología XIX Jornadas de Investigación VIII Encuentro de Investigadores en Psicología del MERCOSUR. Facultad de Psicología - Universidad de Buenos Aires, Buenos Aires.

Organización Panamericana de la Salud. (2012). Por la salud de las personas trans. Elementos para el desarrollo de la atención integral de las personas trans y sus comunidades en Latinoamérica y El Caribe. México.

Orozco Calderón, Gabriela y Ostrosky Shejet, Feggy y Salin Pascual, Rafael y Borja Jiménez, Karina (2011). Perfil neuropsicológico en transexuales: efecto de reasignación del sexo. Revista Chilena de Neuropsicología, 6 (1), 34-41.

Pan, B., Lu , Y., Wilson, A., Jianjun, O., \& Chen, R. (2020). Health care and mental health challenges for transgender individuals during the COVID-19 pandemic. The Lancet Diabetes \& Endocrinology(8). doi:10.1016/S2213-8587(20)30182-0.

Parlamento Europeo (2011). "Resolución del Parlamento Europeo, de 28 de septiembre de 2011, sobre derechos humanos, orientación sexual e identidad de género en las Naciones Unidas".

Serret, E. (2009). La conformación reflexiva de las identidades transexuales. Sociológica, 24(69), 79 - 100. 
Smith J.A, Flores P, Larkin M. (2009) (2009). Interpretative phenomenological analysis: Theory, method and research. London. Sage Publications.

Su, D., J. A., Fisher, C., Ramos, A., Kelley, M., Rogel Mendoza, D. A. \& Coleman, J. D. (2016). Mental health disparities within the LGBT population: A comparison between transgender and nontransgender individuals. Transgender Health, 1, 12-20. https://doi.org/10.1089/trgh.2015.0001

Swaab, D. F. (2004). Sexual differentiation of the human brain: Relevance for gender identity, transsexualism and sexual orientation. Gynecol Endocrinol, 19, 301-312. http://dx.doi.org/10.1016\%2F0166-2236\%2895\%2980007-O

Williams R.W, Roberts, G.W., Irvine, F.E.\& Hastings, R. (2010). Exploring decision making in intellectual disability nursing practice. A qualitative study. Journal of Intellectual Disabilities 14, no. 3: 197-220. 